\title{
Invasive Fungal Laryngitis in an Immunocompetent Person
}

\author{
${ }^{1}$ Gautam Khaund, ${ }^{2}$ Debika Baruah, ${ }^{3}$ Vivek Agarwal, ${ }^{4}$ Surajit Barman
}

\begin{abstract}
Fungal laryngitis is common in immunocompromised patients. But it is rarely seen in a immunocompetent person. Only around 40 cases have been reported in world literature so far. This condition is often confused with premalignant conditions like leukoplakia, dysplasia. Our patient, a 35 years old immunocompetent male, presented with hoarseness, who later on developed stridor and had to be tracheostomized. Laryngoscopy was done under general anesthesia and biopsy taken. Histopathological examination revealed fungal laryngitis (Aspergillus). The patient recovered completely after antifungal treatment.
\end{abstract}

Keywords: Invasive fungal laryngitis, Immunocompetent person, Laryngitis, Fungal laryngitis.

How to cite this article: Khaund G, Baruah D, Agarwal V, Barman S. Invasive Fungal Laryngitis in an Immunocompetent Person. Int J Phonosurg Laryngol 2014;4(2):73-75.

\section{Source of support: Nil}

Conflict of interest: None

\section{INTRODUCTION}

Fungal laryngitis is often seen in immunocompromised patients but is often overlooked in an immunocompetent patient. It is more often than not mistaken for leukoplakia or dysplasia of the vocal folds. Around 40 cases of fungal laryngitis have been reported in immunocompetent persons in world literature. ${ }^{1}$ The examiner needs to have a sufficient degree of suspicion in order to diagnose fungal laryngitis (in nonimmunocompromised), which could be superficial or invasive.

\section{CASE HISTORY}

Mr RS, a 35-year-old man presented with the main complaint of hoarseness for the last 2 months and dyspnea on exertion for last 2 weeks. He was examined in the OPD and the only finding was white patches on both the vocal folds. The vocal folds were also edematous. We thought of differential diagnosis of leukoplakia, granulomatous conditions and fungal infections. The patient was investigated. There was no abnormality in the blood picture. The

\footnotetext{
${ }^{1}$ Chief Consultant, ${ }^{2-4}$ Consultant

${ }^{1-4}$ Department of ENT, Nightingale Hospital, Guwahati Assam, India

Corresponding Author: Gautam Khaund, Chief Consultant Department of ENT, Nightingale Hospital, Guwahati-781005 Assam, India, e-mail: gautamkhaund@rediffmail.com
}

chest X-ray was normal. Computed tomography scan of the larynx demonstrated only thickened vocal folds and no other abnormality.

The patient was advised video laryngeal surgery (VLS). But the patient refused surgery and went home. After a period of 15 days, he came back to the hospital in stridor/? respiratory distress. He was tracheostomized. Then VLS was done under general anesthesia.

The white slough like material from the surface of the vocal folds were removed. Underlying soft tissue from the vocal folds were taken for histopathological examination. There was extension of the slough to subglottis which was the probable cause of the increase in respiratory difficulty (Fig. 1).

The histopathological examination report revealed fungal hyphae in the mucosa and submucosa.

The fungus was identified as Aspergillus.

The patient was treated with antifungals. Initially, we started with IV Voriconazole at the dose of $200 \mathrm{mg}$ twice
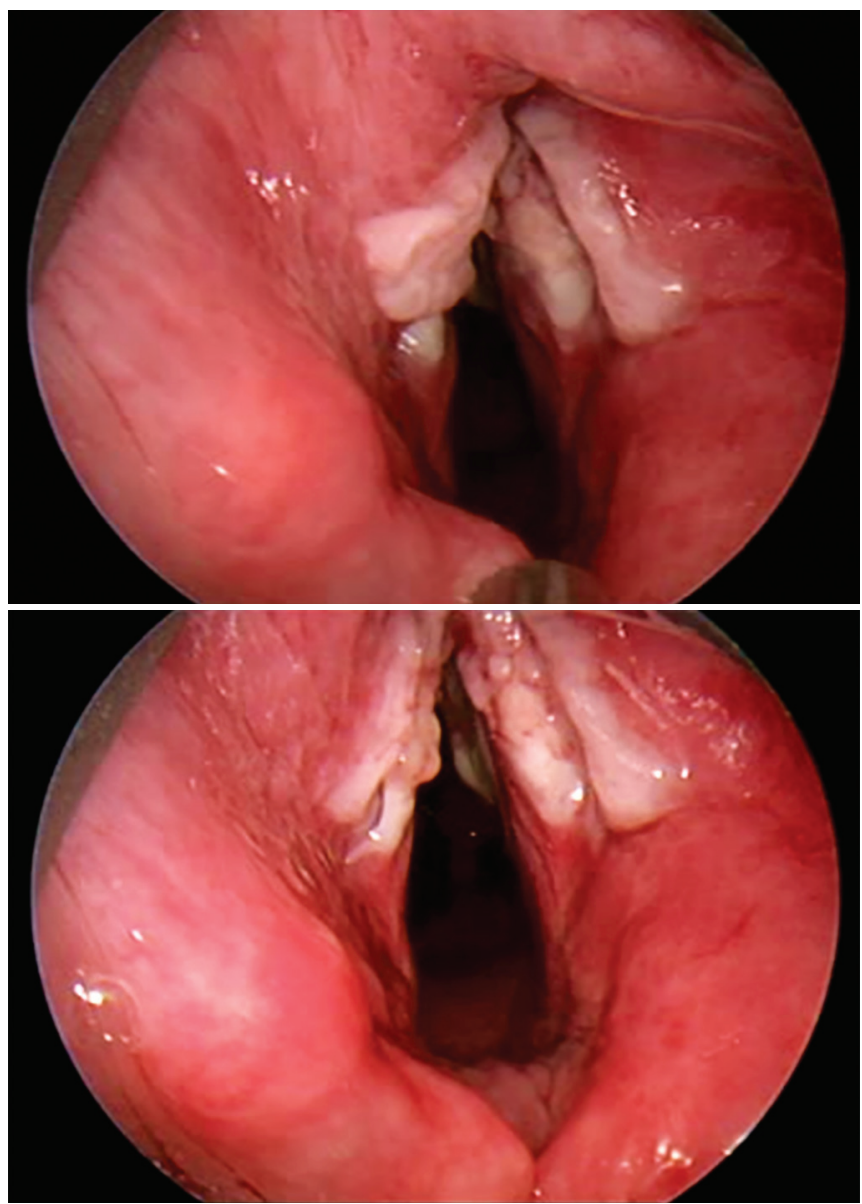

Fig. 1: Endoscopic view of the vocal folds covered with a white slough anteriorly and extending to subglottis 


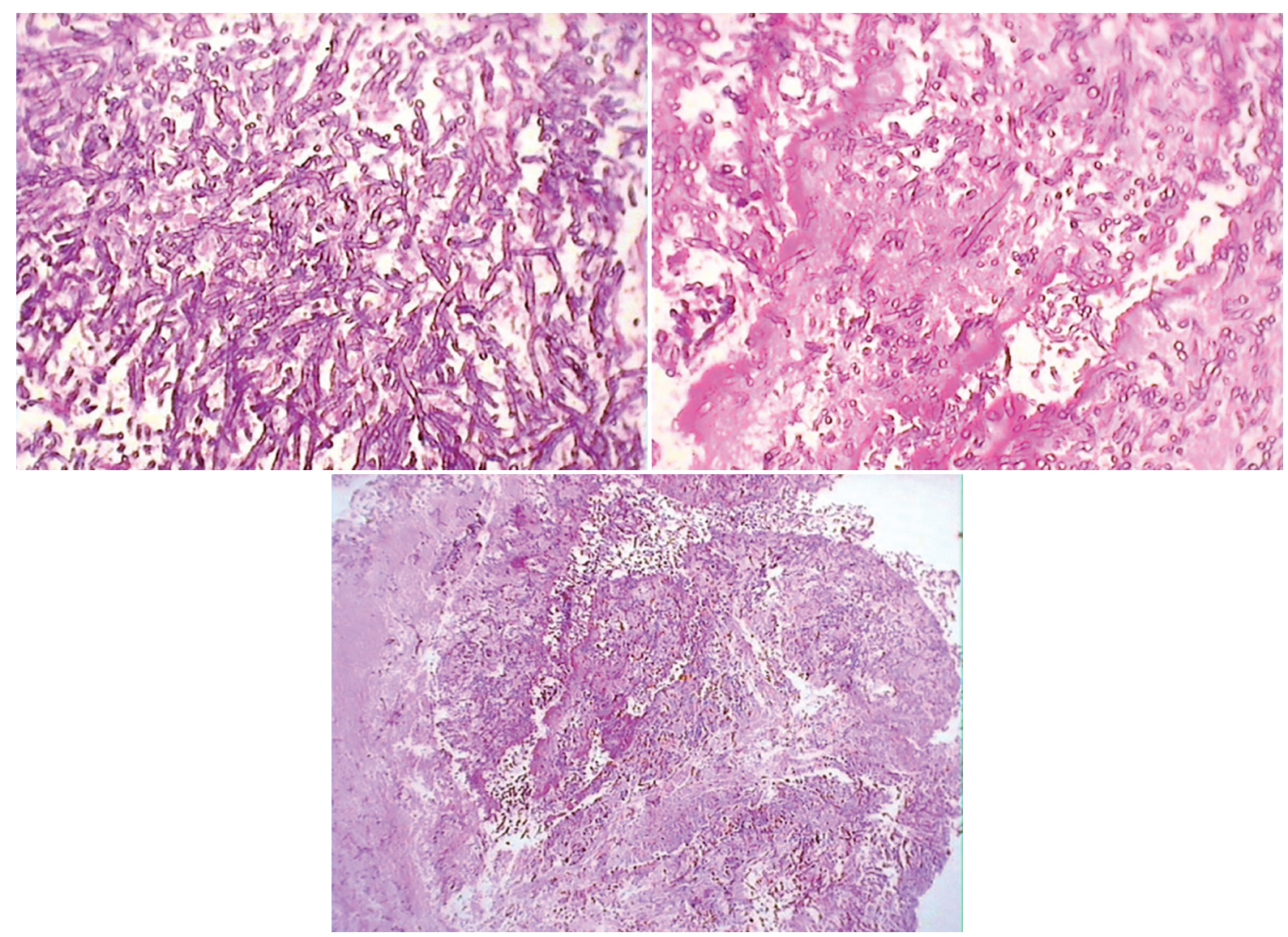

Fig. 2: Photographs of the HPE slides stained with PAS showing fungal hyphae in the submucosa

daily. After 5 days, there was a miraculous improvement. We then converted it to the oral form and sent the patient home with the tracheostomy. His liver function test was monitored. He came back after a month and his larynx on examination looked normal. His tracheostomy was closed. But as he could not afford Voriconazole we converted the antifungal to Itraconazole (200 mg twice daily). He continued antifungals for 3 months. At the end of 3 months (Fig. 3), the patient was symptom free and we stopped all antifungals and have kept him on regular follow-up.

\section{DISCUSSION}

Fungal infection of the larynx is quite common in immunocompromised patients but fungal laryngitis in immunocompetent person is uncommon. In fact, only around 40 cases have been reported in world literature. ${ }^{1,3}$ Various etiological factors have been cited as probable factors promoting fungal infections. Fungal laryngitis is seen in patients using steroid inhalers. Similar presentations could be seen in leukoplakia or smokers dysplasia in immunocompetent hosts. ${ }^{1,2}$ Fungal laryngeal infections can mimic gastroesophageal reflux, granulomatous diseases, leukoplakia and carcinoma. ${ }^{1,4,5}$

Although, mycotic infection can occur in immunocomprised patients, sometimes these infections may be seen in the immunocompetent host in whom there are alterations in the mucosal barrier. ${ }^{1}$

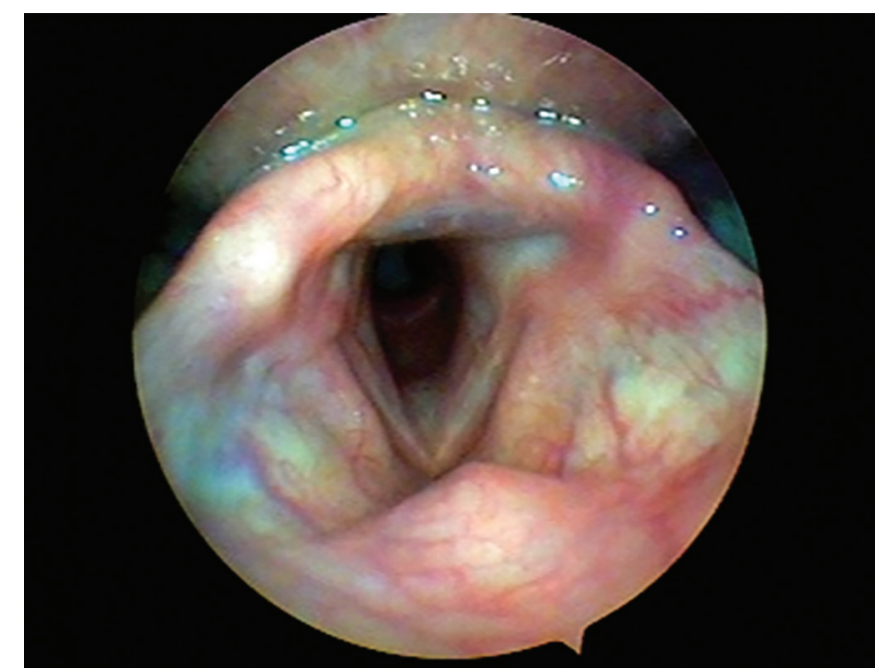

Fig. 3: Post antifungal treatment view of larynx showing normal appearance

The most common fungi causing laryngitis is Candida. ${ }^{1,3}$ Other fungal infections like Aspergillosis, cryptococcal, blastomycosis, histoplasmosis are implicated. All strains of Aspergillus are not tissue invasive. Only those species that grow at $37^{\circ} \mathrm{C}$ are tissue invasive.

A definitive diagnosis is made by the demonstration of fungal spores or hyphae either by tissue biopsy or in $\mathrm{KOH}$ smear. Hematoxylin and eosin (H\&E) stain shows epithelial hyperplasia with hyperkeratosis, neutrophil 
within the upper epithelial layers, lymphocytes, plasma cells and scarring in (Fig. 2) the submucosal stroma. Periodic acid schiff stain brings out the fungal elements better than H\&E stain. Aspergillosis has septate hyphae with acute angle branching in a necrotic lesion.

Treatment of fungal laryngitis is by antifungal medication either orally or intravenous. Voriconazole, itraconazole or posaconazole is administered for 3 months for invasive disease. Voriconazole and itraconazole are administered at a dose of $200 \mathrm{mg}$ twice daily while posaconazole is administered at $400 \mathrm{mg}$ twice daily. Our patient was also put on antifungal medication for 3 months. All these antifungals are effective against $A s-$ pergillus. Cessation of treatment depends on the clinical absence of fungal infection. ${ }^{6}$ At completion of 3 months our patient was disease free clinical and now on regular follow-up without any signs of recurrence.

\section{REFERENCES}

1. Ravikumar A, Kumar P, Somu L, Sudhir B. Fungal laryngitis in immunocompetent patient. Ind J Otolaryngol Head Neck Surg 2014 Jan;66(Suppl):375-378.

2. Liu YC, Zhou,Ling L. Aetiological factors contributing to the development of primary laryngeal aspergillosis in immunocompetent patient. J Med Microbiology 2010 Oct;59(Pt 10): 1250-1253.

3. Mehanna HM, Kuo T, Chaplin J, Taylor G, Morton RP. Fungal laryngitis in immunocompetent patient. J Laryngol Otol 2004 May;118(5):379-381.

4. Nunes FB, Bishop T, Prasad ML, Madison JM, Kim DY. Laryngeal candidiasis mimicking malignancy. Laryngoscope 2008 Nov 1;18(11):1957-1959.

5. Scheid Sara C, Anderson Timothy D, Sataloff Robert T. Ulcerative fungal laryngitis. Laryngoscopic Clinic. Ear Nose Throat J 2003;82:168.

6. Denning DW. Harrison's principles of internal medicine, 18th ed, volume 1, part 8: Infectious Disease, section 16: fungal infection, Chapter 204 Aspergillosis 1659. 